\title{
A rapid method for protein staining in polyacrylamide gels using water saturated with chloroform
}

\author{
Paweł P. Pięta ${ }^{1 凶}$, Ewa Burchacka2* ${ }^{2}$, Aleksandra Śliwa ${ }^{3 *}$ and Anna Szczerba ${ }^{3}$ \\ 'Department of Bionic and Experimental Medical Biology, Poznan University of Medical Sciences, Poznań, Poland; 2 Department of Medical and \\ Organic Chemistry, and Microbiology, Faculty od of Chemistry, Wroclaw University of Science and Technology, Wrocław, Poland; ${ }^{3}$ Cell Biology \\ Department, Poznan University of Medical Sciences, Poznań, Poland
}

Polyacrylamide gel electrophoresis, followed by an appropriate staining, is a popular and useful analytical procedure for protein identification and characterization. The aim of this study was to develop a method for protein visualization in polyacrylamide gels that would be alternative to Coomassie blue or silver staining. The proposed method is simple, fast and inexpensive. The optimized protocol for protein staining and visualization takes as little as 6 minutes and utilizes deionized water and chloroform. Fluorescence of proteins is induced by UV light and can be detected with a standard transilluminator.

Keywords: PAGE, gel imaging, chloroform, protein staining

Received: 24 April, 2021; revised: 26 April, 2021; accepted: 04 May, 2021; available on-line: 11 May, 2021

๑e-mail: pawel.pieta@ump.edu.pl

*These authors contributed equally to this work

Acknowledgements of Financial Support: This research was funded by the Statute Funds 2783 of the Poznan University of Medica Sciences (PP) and Statute Funds 8211104160 of the University of Science and Technology (EB).

Abbreviations: Au, arbitrary units; PAA, polyacrylamide; PAGE, polyacrylamide gel electrophoresis

\section{INTRODUCTION}

Polyacrylamide gel electrophoresis (PAGE) is a very popular analytical technique used for numerous biological applications. PAGE, followed by a specific staining protocol, is a gold standard particularly in protein analysis, including determination of protein molecular weight, purity and expression level (Laemmli, 1970; Rolf et al., 2019; Gulamhussein et al., 2020).

Among many protocols for protein visualization in gels, the most popular involve the use of dyes, such as colloidal Coomassie blue and silver nitrate. All of these protocols are routinely used in research laboratories and even though they are declared as rapid, they are in fact time consuming as the staining procedure usually takes more than 3 hours (Winkler et al., 2007; Sasse and Gallagher, 2009).

A good alternative for these conventional staining protocols are ready to use precast gels supplemented with a trihalo compound or polyacrylamide mixes supplemented with the same reagent. These seem to be a good choice for fast gel visualization (Gürtler et al., 2013).

The physical basis of protein visualization with trihalo compouds was described by Kazimin and co-workers in 2002 (Kazmin et al., 2002). Detection is possible thanks to the light-induced reactions of tryptophan residues of the analyzed proteins with the halo compounds, such as the trichloroethan, trichloroethanol, bromoform and chloroform. The results of the reaction may be examined using fluorescence detection, NMR-spectroscopy and reverse phase chromatography (Edwards et al., 2002). Analysis of the fluorescence excitation and emission wavelengths demonstrated differences related to the trihalo compounds' structure. Moreover, relative fluorescence intensity of chloroform and trichloroethane is higher than the values characterizing trichloroethanol and bromoform (Edwards et al., 2002).

In 2004, Ladner and coworkers developed and demonstrated high compatibility of protein staining procedure using trihalo compounds (Ladner et al., 2004). This procedure may be used in numerous bioanalytical techniques, such as Western blot or isoelectric focusing.

A protocol with trichloroethanol added to a gel as a staining agent is now a commonly used technique for protein visualization in polyacrylamide gels and related procedures (Ladner et al., 2006; Gürtler et al., 2013).

Trihalo compounds are also utilized by many chemical manufacturers as an integral part of ready to use precast gels or polyacrylamide formulations.

As an alternative to these commercially available methods, we have developed a rapid, simple and costeffective staining protocol for protein visualization in polyacrylamide gels. The proposed protocol for protein staining is based on the use of water supplemented with chloroform, and fluorescence of proteins induced by UV light can be detected with a standard transilluminator.

\section{MATERIALS AND METHODS}

\section{Materials}

Chemicals used for the gel, electrophoresis buffers and sample buffers' preparation, such as glycine, Trisbase, sodium dodecyl sulfate (SDS), acrylamides mixture 37.5:1, ammonium persulfate (APS), and beta-mercaptoethanol were purchased from Bioshop (Canada). Reagents used for preparation of the staining solutions, such as chloroform, methanol, Coomassie brilliant blue, and acetic acid were purchased from Merck (Germany). Tryptophan containing proteins characterized in Table 1 were provided as a kind gift by Pure Biologics S.A. (Poland).

\section{Methods}

Protein separation by SDS-PAGE. Protein samples were analyzed by means of standard Laemmli method using the Mini-Protean Tetra Cell for 1-D vertical gel electrophoresis (Bio-Rad) (Laemmli, 1970). Protein samples were mixed with $4 \times$ Laemmli sample buffer, dena- 
Table 1. Characterization of tryptophan containing proteins

\begin{tabular}{llll}
\hline Protein & $\begin{array}{l}\text { Number of trypto- } \\
\text { phan residues }\end{array}$ & $\begin{array}{l}\text { Molecular } \\
\text { weight } \\
\text { [Da] }\end{array}$ & $\begin{array}{l}\text { Content of } \\
\text { tryptophan \% }\end{array}$ \\
\hline $\mathrm{Pb0001}$ & 1 & 18320 & 1.11 \\
\hline $\mathrm{Pb0002}$ & 1 & 21586 & 0.95 \\
\hline $\mathrm{Pb0003}$ & 4 & 26034 & 3.13 \\
\hline $\mathrm{Pb} 0004$ & 11 & 39311 & 5.71 \\
\hline
\end{tabular}

tured at $90^{\circ} \mathrm{C}$ for 10 minutes and subsequently cooled on ice. Each sample was loaded into a separate well. Electrophoretic separation of proteins was performed using a $4 \%$ stacking gel and a $15 \%$ separating gel under the following conditions: $35 \mathrm{~mA}$ for 15 minutes, and $55 \mathrm{~mA}$ for 20 minutes (Laemmli, 1970).

Preparation of the chloroform staining solution. $100 \mathrm{ml}$ of deionized water were combined with $0.5 \mathrm{ml}$ of chloroform and vigorously mixed by vortexing for 60 seconds. The obtained solution was stored at room temperature and protected from light until further use.

Optimization of the protein staining protocol. The protocol was developed by analysis of gel slices which contained $500 \mathrm{ng}$ of tryptophan containing protein mix- tures. Slices were immersed in the chloroform staining solution and incubated at room temperature for 1 , 3 and 5 minutes, respectively. After incubation, the gel slices were placed on the surface of a UV transilluminator (component of the Chemidoc XR+ imaging system manufactured by Bio-Rad). Gel images were recorded after 1, 2, 3, 4 and 5 minutes of exposure to UV radiation. Results were analyzed by using the ImageLab software (Bio-Rad).

Detection limit for the chloroform staining solution. In order to assess the detection limit of the staining protocol, samples containing $1000 \mathrm{ng}$, $500 \mathrm{ng}$, $250 \mathrm{ng}, 125 \mathrm{ng}$, and $62.5 \mathrm{ng}$ of each protein were analyzed. Samples were separated as described in section "Protein separation by SDS-PAGE". Gels were stained by incubation in the chloroform staining solution for 3 minutes and exposed to UV radiation for 3 minutes. Gel images were analyzed with the ImageLab software.

Protein polyacrylamide gel staining with Coomassie brilliant blue. The developed chloroform staining protocol was compared with the commonly used Coomassie staining procedure. Proteins separated in polyacrylamide gels were stained using Coomassie brilliant blue as described by Sasse and Gallagher (Sasse \& Gallagher, 2009).

Data analysis. The recorded results were analyzed by using the Image Lab software (Bio-Rad). Signal intensities were measured using a densitometric method ac-
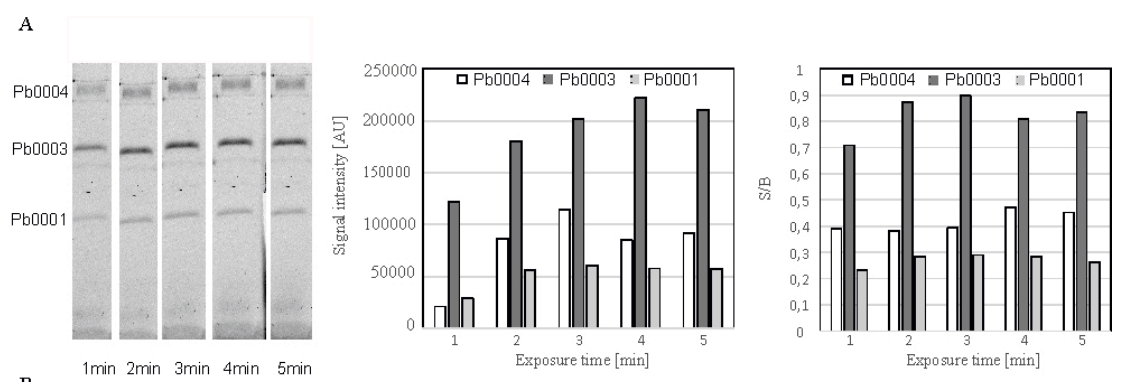

B
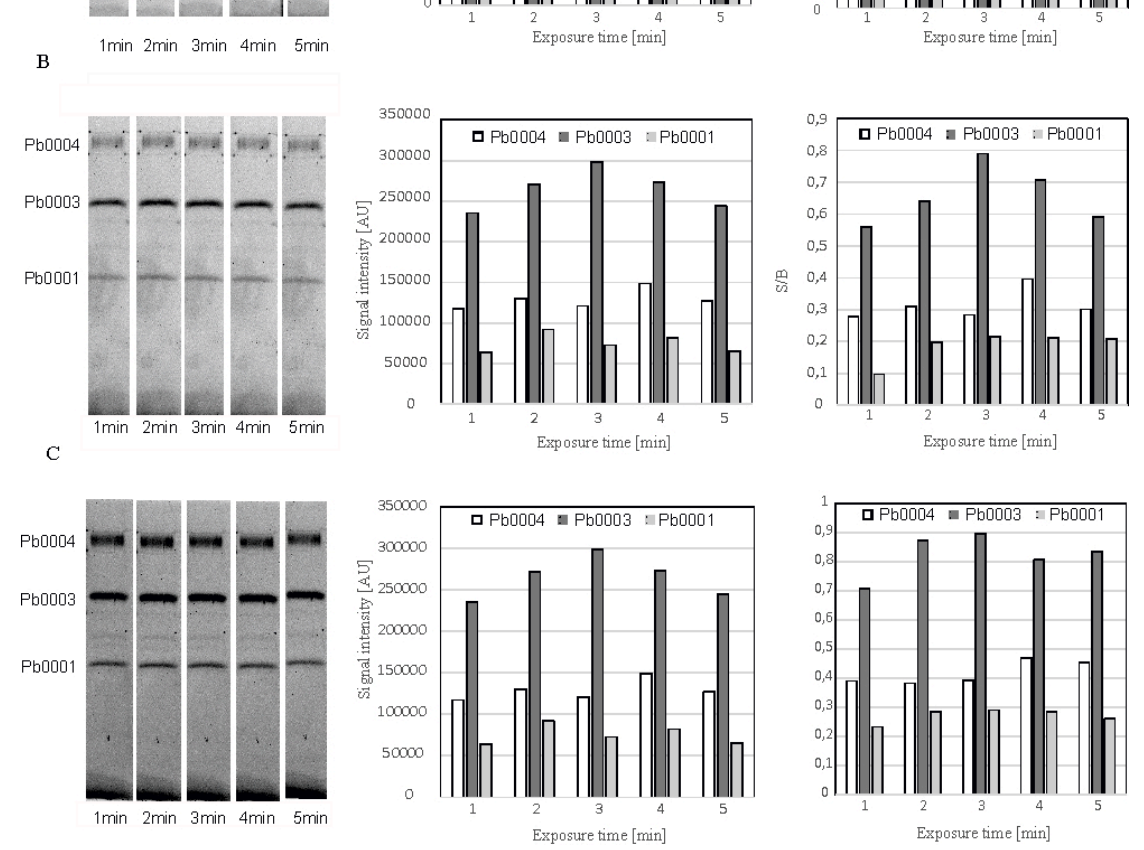

Figure 1. Chloroform staining and protein ( $\mathrm{Pb001}, \mathrm{Pb003}$ and $\mathrm{Pb004)}$ visualization in the polyacrylamide gel.

A - Gel incubation for 1 min in water saturated with chloroform; detection after 1, 2, 3, 4, 5 min of exposure to UV. Left panel - gel visualization, central panel - comparison of signal intensities, right panel - comparison of signal to background ratios. B - Gel incubation for 3 min in water saturated with chloroform; detection after 1, 2, 3, 4, 5 min of exposure to UV. Left panel - gel visualization, central panel - comparison of signal intensities, right panel comparison of signal to background ratios. C - Gel incubation for 5 min in water saturated with chloroform; detection after 1, 2, 3, 4, 5 min of exposure to UV. Left panel - gel visualization, central panel - comparison of signal intensities, right panel comparison of signal to background ratios. 
cording to the size of the bands, while the background was measured individually for each band. Signal to background ratio was calculated and used for data presentation.

All experiments and measurements were made in duplicates.

\section{RESULTS AND DISCUSSION}

The aim of this study was to develop a fast and simple protocol for protein visualization in polyacrylamide gels by using chloroform as a staining agent.

In the first step of the procedure, the optimal time of gel incubation in chloroform saturated water and subsequent gel exposure to UV radiation was verified. For this purpose, gel fragments containing a mixture of $500 \mathrm{ng}$ of proteins (Pb001, Pb003 and Pb004) were stained with chloroform water and exposed to UV. The results showed that already after 1 minute of gel incubation with chloroform water it is possible to observe weak signals indicating the proteins' presence (Fig. 1A). The signal intensities were in the range between 3640208260 arbitrary units (Au). However, the optimal time of gel staining for further protein visualization was 3 minutes (Fig. 1B); after this time, the signal intensities were in the range between 7350-328953 Au. Five minute incubation of the gel in chloroform water resulted in much stronger signals, with intensities in the range be-

A

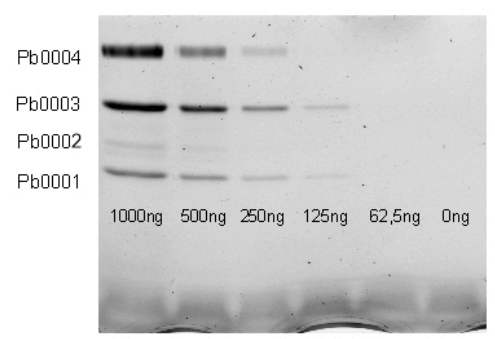

C

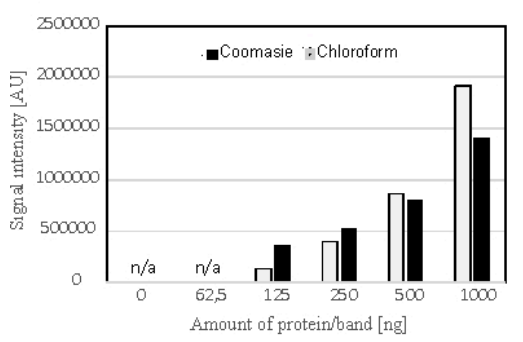

E

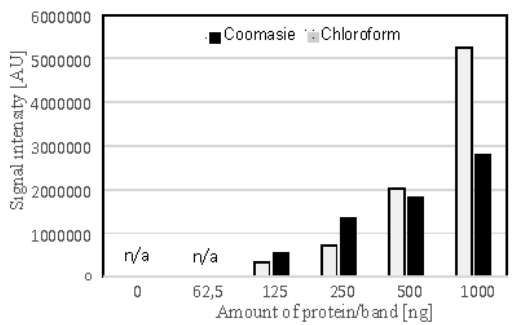

tween 14388-517160 Au, as shown in Fig. 1C. Still, the increased incubation time resulted in strong background and appearance of some artefacts, which might be breakdown products of the originally intact proteins (Fig. 1C).

With regards to the time of UV irradiation, it was shown that even 1 minute of gel exposure allows for visualization of the protein bands. However, the optimal time for protein detection was established to be $3 \mathrm{~min}-$ utes (Fig. 1). After this time, both the strongest signals, as well as the best ratio of signal to background noise was observed.

The results of gel visual, as well as densitometric analysis of the obtained pictures confirmed that the method employing chloroform allows for effective protein staining. The proposed protocol consists of two steps: (1) gel incubation with water supplemented with chloroform (ratio: $0.5 \mathrm{ml}$ chloroform : $99.5 \mathrm{ml}$ deionized water) for 3 minutes, (2) gel irradiation with UV light for $3 \mathrm{~min}$ utes, followed by visualization.

This protocol was further used for determination of the protein detection limit. For this purpose, $1000 \mathrm{ng}$, $500 \mathrm{ng}, 250 \mathrm{ng}, 125 \mathrm{ng}$, and $62.5 \mathrm{ng}$ of the analyzed proteins were separated in a PAA gel, stained and visualized. The experiments demonstrated that the chloroform staining is useful for protein detection in the range of $125 \mathrm{ng}$ to $500 \mathrm{ng}$ (Fig. 2A). This detection limit is related to the protein molecular weight and tryptophan content in the polypeptide chain. The lowest detection

B

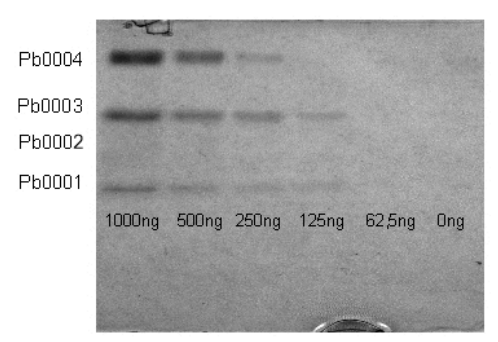

$\mathrm{D}$

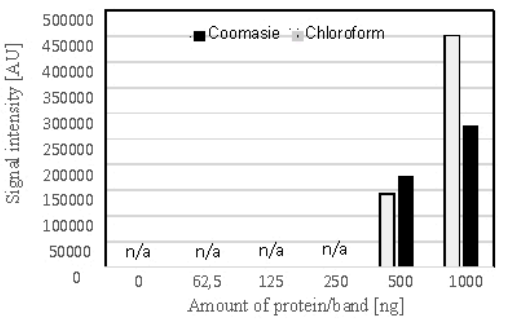

F

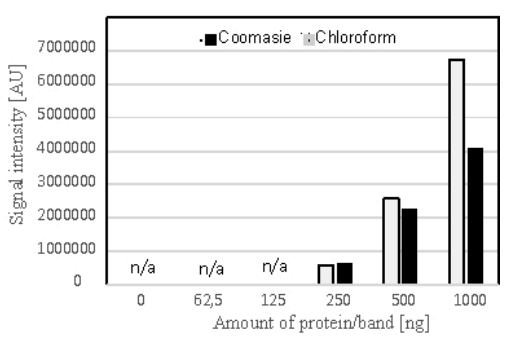

Figure 2. Results of protein staining showing detection limits for the two methods: staining with water saturated with chloroform, and the Coomassie method.

A - Detection limit for proteins stained with water saturated with chloroform, B - Detection limit for proteins stained with the Coomassie method. C - Relationship between the signal intensity and protein amount for the Pb0001 protein (MW 18.320 kDa, \%W 1.11), D - Relationship between signal intensity and protein amount for the Pb0002 protein (MW 21.586 kDa, \%W 0.95), E - Relationship between signal intensity and protein amount for the Pb0003 protein (MW $26.034 \mathrm{kDa}, \% \mathrm{~W} 3.13$ ), F - Relationship between signal intensity and protein amount for the PB0004 protein (MW $39.311 \mathrm{kDa}$ \% $\%$ 5.71). 
<smiles>[R]C1=c2[nH]c3ccccc3c([2H])c[nH]c2=C1[2H]</smiles>

Figure 3. Schematic represenatation of reaction between indole (tryptophane residue) and chloroform.

limit equal to $125 \mathrm{ng}$ was obtained for proteins: Pb0001 (MW $18.320 \mathrm{kDa}$ ) and Pb0003 (26.034kDa), containing $75 \mathrm{fmol}$ and $150 \mathrm{fmol}$ of tryptophan, respectively. In case of the $\mathrm{Pb} 0004$ protein (MW 39.013kDa), with tryptophan content equal to $230 \mathrm{fmol}$, the detection limit was established at $250 \mathrm{ng}$. The highest detection limit, close to $500 \mathrm{ng}$, was noted for the Pb0002 protein (MW $21.586 \mathrm{kDa}$ ) with tryptophan content of $220 \mathrm{fmol}$. The differences in the detection limits of particular proteins might be the result of different accessibility of tryptophan during the reaction.

The results of the experiments conducted with chloroform as a staining agent were also compared with the standard and most commonly used protocol of protein visualization of the gel using Coomassie brilliant blue. The detection limits for proteins Pb0001, Pb0003 and $\mathrm{Pb} 0004$ obtained with the Coomassie agent were similar to those obtained by using the chloroform staining method. However, in case of the Pb0002 protein, the chloroform protocol was more sensitive in comparison to the classic Coomassie method (Fig. 2). Higher sensitivity of the developed protocol might be related to the protein amino acid composition.

Protein staining using chloroform occurs due to a reaction between chloroform and tryptophan residues, as shown in Fig. 3. On the other hand, the Coomassie dye binds to proteins through ionic interactions with positively charged amino acid residues (arginine, lysine), and van der Waals interactions with tyrosine (de Moreno, Smith and Smith, 1986).

As mentioned earlier, the lowest detection limit range of our protocol is between 125-500 $\mathrm{ng}$ and depends on the protein molecular weight and tryptophan content.

The results of the experiments which showed chloroform as a sensitive protein staining agent, are in agreement with a study previously published by Ladner and coworkers (Ladner et al., 2004). Still, the detection limit of fluorescent visualization of proteins by trichloroethanol in the Ladner's method was established as $250 \mathrm{ng}$ of protein, and in our case the lowest level of protein detected after chloroform staining was $125 \mathrm{ng}$.

Common availability of chloroform in all biochemical laboratories makes the proposed method a good alternative for trichloroethanol based protocols, including ready to use stain-free gels recommended for routine applications. This method can be used for staining of any proteins containing tryptophan.

One of the main advantages of chloroform staining is also the fact that the whole procedure, including protein staining and visualization, lasts only 6 minutes. It is worth mentioning that the proposed protocol does not require any changes in the gel casting process, which might be a critical aspect for various research groups.

We are aware of the fact that using pure chloroform on the transilluminator surface is not recommended by any equipment manufacturer because it is related to irreversible changes on the transilluminator surface. Still, the water supplemented with chloroform solution that we propose for staining seems to be safe for the transilluminator surface.

In conclusion, the proposed method has many advantages, including high sensitivity - comparable to the Coomassie blue staining, low costs and short time of the whole procedure. The chloroform staining method is also a good alternative for trichloroethanol-based protocols. Even though the protocol requires to use proteins containing tryptophan, we believe that in time it will be commonly used for standard electrophoresis based procedures, such as protein characterization by means of 2D electrophoresis, analysis of protein transfer during western blotting and other laboratory procedures that require protein gel staining (Ladner et al., 2004; Gürtler et al., 2013; Chopra, Willmore \& Biggar, 2019).

\section{CONCLUSIONS}

This study demonstrates a fast fluorescent technique for protein visualization in polyacrylamide gels where protein fluorescence is related to tryptophan photo-modification by chloroform. The protein chloroform staining protocol consist of two steps: 1) incubation of the gel with separated proteins in $0.5 \%$ water chloroform solution for 3 min, followed by 2) gel irradiation with UV for 3 minutes. The developed protocol is simple, fast and inexpensive.

The protein staining procedure using water saturated with chloroform presents a good alternative for commonly used methods based on trichloroethanol (Edwards et al., 2002; Kazmin et al., 2002).

\section{Author Contributions}

PP designed the study, performed experiments, wrote the manuscript. EB and AS analyzed the results and performed interpretation of the data and drafted the manuscript. AS interpreted data and wrote the manuscript. All authors read and approved the final manuscript.

\section{Data Availability Statement}

Data are available at: http://www.katbiolkom.ump. edu.pl/wp-content/uploads /2021/repository/rapid_ method.zip

\section{Acknowledgments}

The authors would like to thank to Dr. Robert Kołodziejczyk and MSc eng. Ewa Margas from Pure Biologics S.A. (Wrocław, Poland) for providing tryptophan containing proteins used for the protocol development.

\section{Conflicts of Interest}

The authors declare no conflict of interest.

\section{REFERENCES}

Chopra A, Willmore WG, Biggar KK (2019) Protein quantification and visualization via ultraviolet-dependent labeling with 2,2,2-trichloroethanol. Sci Rep 9: 1-8. https://doi.org/10.1038/s41598-019-50385-

Edwards RA, Jickling G, Turner RJ (2002) The light-induced reactions of tryptophan with halocompounds. Photochem Photobiol 75: 362. https://doi.org/10.1562/0031-8655(2002)075<0362:tlirot $>2.0 . c 0 ; 2$

Gulamhussein AA, Uddina R, Tigheb BJ, Poynera DR, Rothniea AJ (2020) A comparison of SMA (styrene maleic acid) and DIBMA (di-isobutylene maleic acid) for membrane protein purification. Biochim Biophys Acta (BBA) - Biomembranes 1862: 183281. https://doi. org/10.1016/j.bbamem.2020.183281

Gürtler A, Kunz N, Gomolka M, Hornhardt S, Friedl AA, McDonald K, Kohn JE, Posch A (2013) Stain-Free technology as a nor- 
malization tool in Western blot analysis. Anal Biochem 433: 105-111. https://doi.org/10.1016/j.ab.2012.10.010

Kazmin D, Edwards RA, Turner RJ, Larson E, Starkey J (2002) Visualization of proteins in acrylamide gels using ultraviolet illumination. Anal Biochem 301: 91-96. https://doi.org/10.1006/abio.2001.5488

Ladner CL, Yang J, Turner RJ, Edwards RA (2004) Visible fluorescent detection of proteins in polyacrylamide gels without staining. Anal Biochem 326: 13-20. https://doi.org/10.1016/j.ab.2003.10.047

Ladner CL, Edwards RA, Schriemer DC, Turner RJ (2006) Identification of trichloroethanol visualized proteins from two-dimensional polyacrylamide gels by mass spectrometry. Anal Chem 78: 23882396. https://doi.org/10.1021/ac051851y

Laemmli UK (1970) Cleavage of structural proteins during the assembly of the head of bacteriophage T4. Nature 227: 680-685. https:// doi.org/10.1038/227680a0 de Moreno MR, Smith JF, Smith RV (1986) Mechanism studies of coomassie blue and silver staining of proteins. J Pharm Sci 75: $907-$ 911. https://doi.org/10.1002/jps.2600750919

Rolf J, Siedentop R, Lütz S, Rosenthal K (2019) Screening and identification of novel cGAS homologues using a combination of in vitro and in vivo protein synthesis. Int J Mol Sci 21: 105. https://doi. org/10.3390/ijms21010105

Sasse J, Gallagher SR (2009) Staining proteins in gels. Curr Protoc Mol Biol 85: 10.6. https://doi.org/10.1002/0471142727.mb1006s85

Winkler C, Denker K, Wortelkamp S, Sickmann A (2007) Silverand Coomassie-staining protocols: Detection limits and compatibility with ESI MS. Electrophoresis 28: 2095-2099. https://doi. org/10.1002/elps.200600670 